\title{
$T_{c s}$ Degradation of ITER TF Samples due to Fast Current Discharges
}

\author{
Kamil Sedlak, Pierluigi Bruzzone, Boris Stepanov, Neil Mitchell, Vladimir Tronza, Alexander Vostner
}

\begin{abstract}
Direct current (DC) tests performed in the past on the conductor samples of the toroidal field (TF) ITER coils revealed degradation of current sharing temperature, $T_{c s}$. The degradation progresses with repetitive electromagnetic (EM) loading, and also with thermal cycles between $4.5 \mathrm{~K}$ and room temperature. This feature was observed on short samples in SULTAN test facility (EPFLSPC, Switzerland) as well as in TF Insert Coil tests in CSMC test facility (Naka, Japan).

We present three independent observations suggesting that initiation of sample quench followed by a fast current discharge, which normally complements every $I_{c}$ and $T_{\text {cs }}$ test in both SULTAN and CSMC, enhances the $T_{\text {cs }}$ degradation rate. The exact mechanism of this contribution to the degradation remains unidentified.
\end{abstract}

Index Terms - TF coils, conductor testing, degradation, quench.

\section{ITER TF SAMPLE DEGRADATION}

$\mathbf{S}$ EVERAL LARGE cable-in-conduit conductors (CICC) ased on $\mathrm{Nb}_{3} \mathrm{Sn}$ exhibit degradation of the DC performance, typically a drop in $T_{c s}$, when the CICC is exposed to repetitive electromagnetic (EM) load or warm-up-cool-down (WUCD) cycles. The performance degradation was observed also in ITER TF and CS conductor samples [1]-[3]. In the latter case, the problem was mitigated by changing the pitch sequence in the CS cable design [4], [5].

The ITER CICC performance degradation has attracted attention from both experimentalists and theoreticians. The most recent findings and discussion on this topics are summarized in [6], [7] and [8]. In its introductory section, the reference [8] summarizes 38 publications somehow related to the degradation issue.

The degradation due to EM load is induced by repetitive current charging/discharging in the operating magnetic field. The $T_{c s}$ degradation is largest during the first $\sim 10 \mathrm{EM}$ cycles, and gets smaller afterwards. The level of degradation observed in TF conductors with identical layout depends on conductor manufacturer. Practically no degradation is observed in conductors produced in Chepetsk Mechanical Plant, Russian Federation.

To study the degradation due to WUCD cycles is more time consuming, as one thermal cycle lasts several days, compared to a few minutes for the EM cycle. A former study [9] concluded that even though there was a visible degradation observed after the first thermal cycle, the following ten or more

(Corresponding author: Kamil Sedlak.e-mail: kamil.sedlak@psi.ch)

K. Sedlak, P. Bruzzone, and B. Stepanov are with École Polytechnique Fédérale de Lausanne (EPFL), Swiss Plasma Center (SPC), CH-5232 Villigen PSI, Switzerland. additional WUCD cycles, without EM loading between them, did not further deteriorate conductor performance. This might seem to be contradicted by recent TF Insert Coil tests performed in Naka [10], where every subsequent thermal cycle led to a visible $T_{c s}$ degradation. The apparent inconsistency of the two observations is interpreted such that the degradation is present only when WUCD is combined with EM cycling, which was the case in [10] but not in [9].

Very recently, a "conditioning" or "training effect" of TF conductors has been suggested in [6]. The authors claim that the level of ITER TF conductor degradation depends on history of conductor (EM and thermal) cycling, and that it is possible to reduce the level of degradation by a suitable sequence of loading.

We come up with a new phenomenon co-responsible for the sample degradation. We observe that the degradation is enhanced (or accelerated) by the "very fast current dumps" that usually conclude any DC test in both SULTAN test facility [11] and Model Coil test facility in Naka [12], [13]. The reasons for this claim are based on three independent observations presented in sections III-V.

\section{II. $\mathrm{T}_{\mathrm{CS}}$ AND I $_{\mathrm{C}}$ TESTS IN SULTAN}

Before coming to the main topic of the paper, we briefly describe direct current (DC) tests in SULTAN. In the $I_{c}$ run, first the requested field value and temperature are set, and then the current is ramped up slowly $(100 \mathrm{~A} / \mathrm{s})$ until the electric field significantly exceeds the critical electric field traditionally set to $E_{c}=10 \mu \mathrm{V} / \mathrm{m}$. In the $T_{c s}$ run, first current is ramped up in steps to the requested operating value, and then the temperature is slowly increased in steps, again until the electric field significantly exceeds $E_{c}$. A typical $I_{c}$ and $T_{c s}$ run last 30 and 100 minutes, respectively.

Normally, both $I_{c}$ and $T_{c s}$ tests in SULTAN end up with a voltage take-off. Due to the working principle of superconducting transformer delivering current to the sample, which interrupts current whenever the resistive voltage in the sample exceeds $\sim 100 \mathrm{mV}$, the current discharges exponentially to zero very quickly after the quench initiation. A typical current dump of ITER TF SULTAN sample in nominal test conditions

N. Mitchell, V. Tronza and A. Vostner are with with the ITER Organization, 13115 Saint Paul lez Durance, France. 


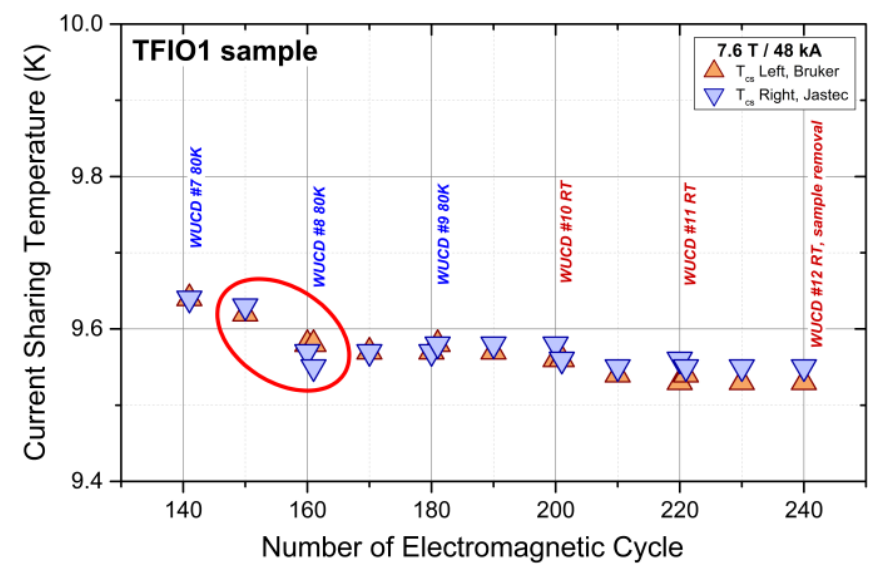

Fig. 1. $T_{c s}$ evolution of TFIO 1 sample. Sample quench happened in the left conductor section (Bruker) at the end of $T_{c s}$ run at 150 EM cycles. Affected is the subsequent $T_{c s}$ at $160 \mathrm{cy}-$ cles. The $T_{c s}$ drops by 40 and $60 \mathrm{mK}$ in the two conductor sections, respectively.

$(10.8 \mathrm{~T}, 68 \mathrm{kA})$ lasts $0.4 \mathrm{~s}$. The temperature in the sample reaches $\sim 30 \mathrm{~K}$. In special cases, if required, the voltage take-off at the end of the SULTAN test can be avoided. For simplicity, we sometimes call the voltage take-off as "sample quench", even though the quench is not fully developed, i.e. it is suppressed in its initial phase by the fast current dump.

\section{MEASUREMENT OF ITER TFIO1 SAMPLE IN SULTAN}

In 2017, ITER Organization launched a dedicated test campaign [6], whose goal was to look for thresholds for the onset of the WUCD-EM degradation and investigate the differences between conductors produced by different manufacturers. The operating field and current were gradually increased during the test campaigns, and many thermal cycles were performed. The results of the test campaign are presented in [6]. Here we present just one particular observation, demonstrated in Fig. 1, which shows the evolution of $T_{c s}$ measured at $7.6 \mathrm{~T}$ and $48 \mathrm{kA}$. This corresponds to $70 \%$ of the nominal operating current and to $50 \%$ of the nominal EM load. After every $10 \mathrm{EM}$ cycles, a $T_{c s}$ measurement was done, and after every three $T_{c s}$ measurements a WUCD cycle, either to $80 \mathrm{~K}$ or to room temperature, was performed.

During the TFIO testing, unlike in the usual SULTAN test campaigns, we tried to avoid the voltage take-off at the end of $T_{c s}$ runs. When the electric field in the sample significantly exceeded the usual criterion of $E_{c}=10 \mu \mathrm{V} / \mathrm{m}$, the sample current was ramped down slowly, with a ramp rate $-300 \mathrm{~A} / \mathrm{s}$ or slower.

The $T_{c s}$ measurement after $150 \mathrm{EM}$ cycles, however, finished with an unintentional voltage take-off and fast discharge, due to the instability of the inlet temperature during the last temperature step of the test. The $T_{\mathrm{cs}}$ measured at $150 \mathrm{EM}$ cycles cannot be influenced by the quench that happened at its very end, but the next $T_{c s}$ measurement, taken at $160 \mathrm{EM}$ cycles could be. And indeed, one can see a drop in $T_{c s}$ at 160 EM cycles in Fig. 1. The $T_{c s}$ drop is observed already before WUCD to $80 \mathrm{~K}$, which was performed after 160 EM cycles. This observation represents the first hint that the performance drop can be a consequence of the preceding voltage take-off followed by the fast current discharge.

\section{ANALYSIS OF THE DEGRADATION ON MANY ITER TF SAMPLES}

Many ITER TF samples have been tested in SULTAN in past years. One of the interesting results of these tests was the $T_{c s}$ evolution along 1000 EM cycles, in which most of the TF samples exhibited DC performance degradation.

The conductor production and testing was divided into four phases. During the initial phases, up to the pre-production phase III, a typical test campaign lasted three weeks, and the number of DC tests (i.e. $T_{\mathrm{cs}}$ and $I_{\mathrm{c}}$ runs) along the EM cycling was higher compared to the final production phase IV lasting just two weeks. This allows us to compare the total drop of $T_{\mathrm{cs}}$ during $1000 \mathrm{EM}$ cycles as a function of the total number of sample quenches between the initial $T_{\mathrm{cs}}\left(T_{\mathrm{cs}} \# 1\right)$ and final $T_{\mathrm{cs}}$ after cycling $\left(T_{\mathrm{cs}} \# 1000\right)$ performed before any WUCD. Typically, $7 \mathrm{DC}$ tests were done between the first and last $T_{\mathrm{cs}}$ measurement in the phase III campaigns, compared to only 4 DC tests for samples in the phase IV. Every DC test ended up by a sample quench.

The corresponding comparison is shown in Fig. 2. The $T_{c s}$ performance drop, expressed as

$$
\Delta T_{c s}=T_{c s} \# 1000-T_{c s} \# 1
$$

is plotted as a function of the number of sample quenches between the $T_{c s} \# 1$ and $T_{c s} \# 1000$. There seems to be a trend in the TF samples for the most manufactures - the more DC tests (sample quenches) were performed between the first and $1000^{\text {th }}$ EM cycle, the larger is the $T_{c s}$ degradation. The trend is not visible in CNTF and USTF samples, presumably due to low statistics of measurements in phase III, and in RFTF samples, in which $T_{c s}$ improves with EM cycling instead of degrading.

The trend present in the data represents a second hint that the sample quenches enhance the observed $T_{c s}$ degradation. 

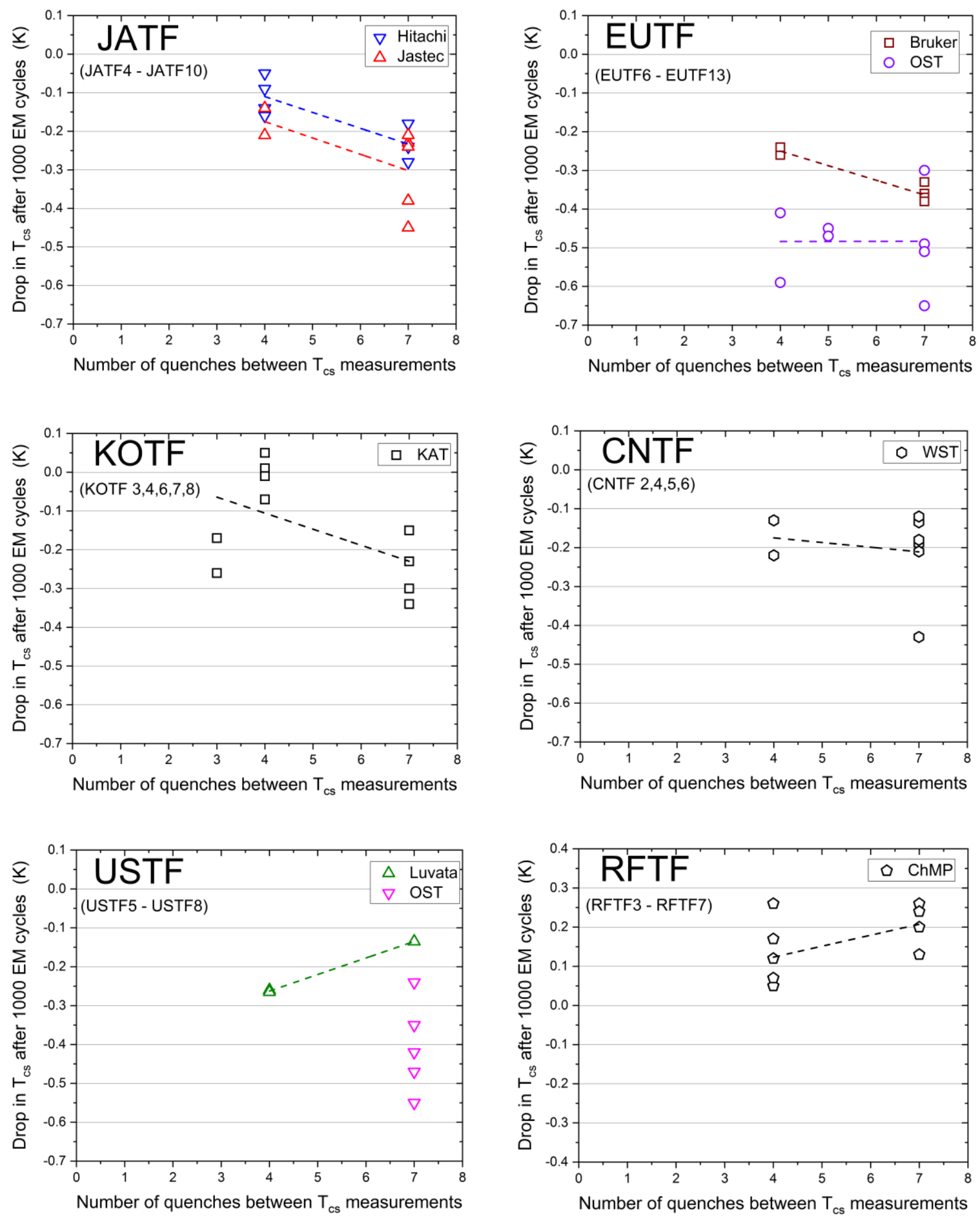

Fig. $2 T_{c s}$ performance drop after $1000 \mathrm{EM}$ cycles $\left(\Delta T_{c s}=T_{c s} \# 1000-T_{c s} \# 1\right)$ as a function of intermediate DC tests, which is equal to the number of intermediate sample quenches. Note that $T_{\mathrm{cs}}$ of the samples of Russian Federation behaves differently - the DC performance did not degrade but rather improved during EM cyclic loading. The dashed lines indicate linear fits. 


\section{DEDICATED SULTAN SAMPLE STUDIES}

The observations presented in sections III and IV triggered a dedicated study, whose aim was to investigate the effect of sample quenches (fast current discharges) on the $T_{\mathrm{cs}}$ degradation. The study was performed on two "identical" SULTAN samples named TFIO6 and TFIO7. Both samples consist of two conductor sections from the same conductor production batch: the left section Jastec, the right one Bruker, see Table 1. Both Jastec sections were heat-treated together, and so were also the Bruker ones.

Table 1 Conductor sections employed in the SULTAN samples, including ITER ID identifiers.

\begin{tabular}{|c||c|c|}
\hline Sample & Left Section & Right Section \\
\hline TFIO6 & Jastec 81JNC032-2 & Bruker 81EAS022 \\
\hline TFIO7 & Jastec 81JNC032-8 & Bruker 81EAS023 \\
\hline
\end{tabular}

The TFIO6 sample testing started "gently". The initial $T_{\mathrm{cs}}$ run has been done without the usual sample quench at the end of run. The second $T_{\mathrm{cs}}$ test was performed only after 1000 cycles, also without quench. A sequence of another $14 T_{\mathrm{cs}}$ runs has followed, at the later stage intentionally ending with quenches and interleaved with electromagnetic load cycling and one thermal cycle to room temperature. Table 2 summarizes the test sequence and also the measured $T_{\mathrm{cs}}$.

The TFIO7 sample testing started right from the beginning in the usual manner as an ordinary SULTAN sample, i.e. with sample quench followed by the fast current discharge at the very end of every run. Quenches were intentionally induced alternately in the Jastec and Bruker legs by controlling the helium inlet temperature. (The idea was to see if the degradation is linked to the section, where the quench was induced, but no such correlation was found.) Eleven DC tests were done until the $1000^{\text {th }} \mathrm{EM}$ cycle, to be compared to two DC test in TFIO6.

The $T_{\text {cs }}$ for both samples are presented in Fig. 3. The two upper plots show directly $T_{\mathrm{cs}}$ values, separately for Jastec and Bruker conductors, while in the lower plots the values are normalized to the initial $T_{\mathrm{cs}}$. One would expect the initial $T_{\mathrm{cs}}$ being the same in
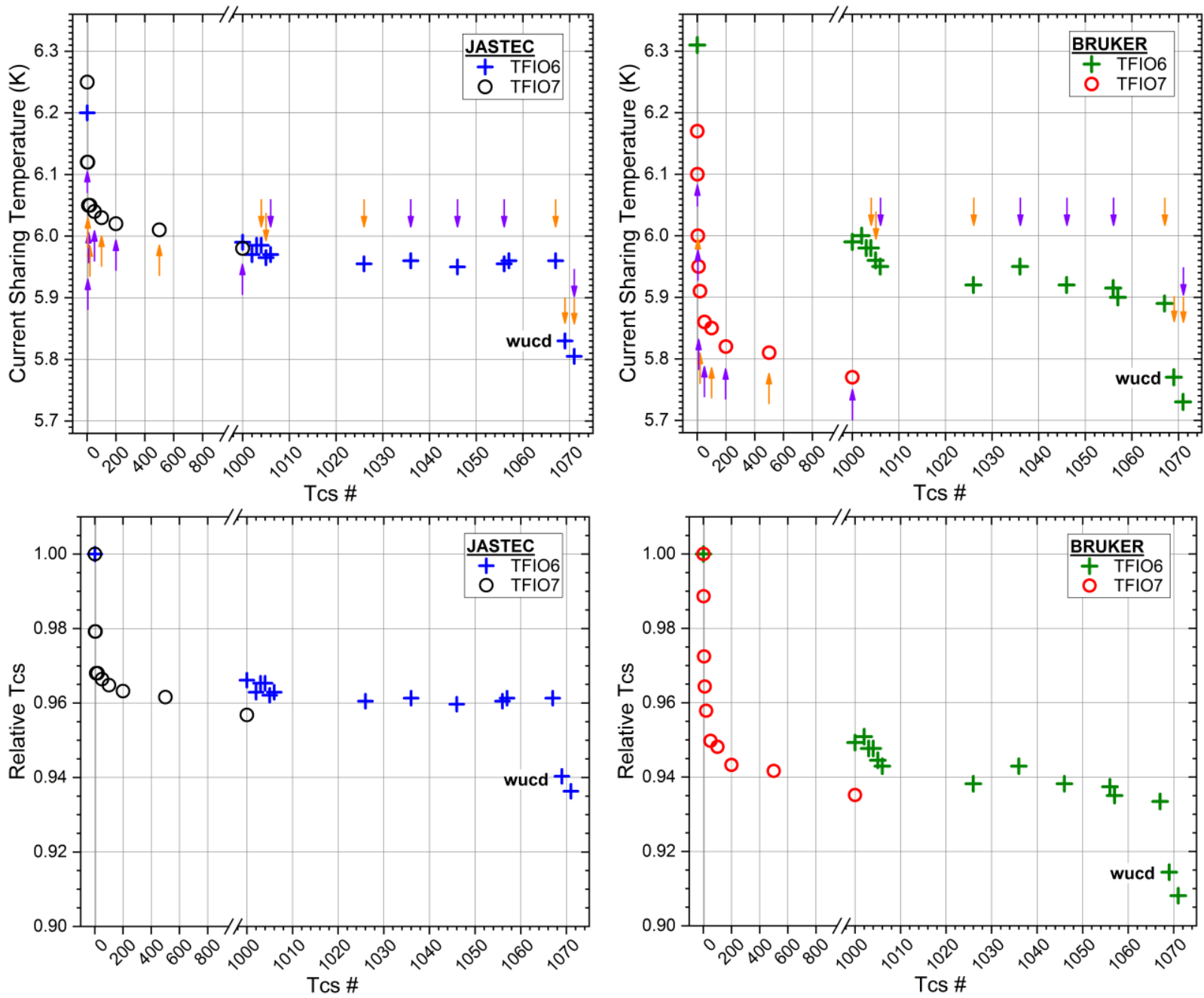

Fig. $3 T_{\mathrm{cs}}$ measured in the TFIO6 and TFIO7 samples (upper plots) and the corresponding relative $T_{\mathrm{cs}}$ normalized to $T_{\mathrm{cs}} \# 1$ (lower plots). The arrows indicate $T_{\mathrm{cs}}$ measurements taken after the previous fast current dump in the Jastec (violet) or Bruker (orange) conductor section. 
both Jastec sections (as well as same in both Bruker sections), however this is not the case, and the differences are $0.05 \mathrm{~K}$ and $0.14 \mathrm{~K}$ for the Jastec and Bruker sections, respectively. Small differences in the initial $T_{\mathrm{cs}}$ of the ITER TF conductors originating from the same manufacturer were observed in the past.

Table 2 List of $T_{\mathrm{cs}}$ runs for the TFIO6 and TFIO7 samples. The comment about quench is related to the quench at the very end of the run, i.e. it may only affect the subsequent test. The two runs with no $T_{\mathrm{cs}}$ values are $I_{\mathrm{c}}$ runs.

\begin{tabular}{|c|c|c|l|}
\hline \multicolumn{4}{|c|}{ TFIO6 sample } \\
\hline Cycle no. & Tcs Jastec & Tcs Bruker & \multicolumn{1}{|c|}{ Comment } \\
\hline \hline 1 & 6.2 & 6.31 & no Quench \\
\hline 1000 & 5.99 & 5.99 & no Quench \\
\hline 1002 & 5.97 & 6 & no Quench \\
\hline 1003 & 5.985 & 5.98 & Quench Bruker \\
\hline 1004 & 5.985 & 5.98 & Quench Bruker \\
\hline 1005 & 5.965 & 5.96 & Quench Jastec \\
\hline 1006 & 5.97 & 5.95 & Quench Bruker \\
\hline 1026 & 5.955 & 5.92 & Quench Jastec \\
\hline 1036 & 5.96 & 5.95 & Quench Jastec \\
\hline 1046 & 5.95 & 5.92 & Quench Jastec \\
\hline 1056 & 5.955 & 5.915 & no Quench \\
\hline 1057 & 5.96 & 5.9 & Quench Bruker \\
\hline 1067 & 5.96 & 5.89 & Quench Bruker, WUCD \\
\hline 1069 & 5.83 & 5.77 & Quench Jastec \\
\hline 1070 & - & - & Quench Bruker \\
\hline 1071 & 5.805 & 5.73 & Quench Jastec \\
\hline
\end{tabular}

\begin{tabular}{|c|c|c|l|}
\hline \multicolumn{4}{|c|}{ TFIO7 sample } \\
\hline Cycle no. & Tcs Jastec & Tcs Bruker & \multicolumn{1}{|c|}{ Comment } \\
\hline \hline 1 & 6.25 & 6.17 & Quench Jastec \\
\hline 2 & 6.12 & 6.1 & Quench Bruker \\
\hline 3 & - & - & Quench Jastec \& Bruker \\
\hline 5 & 6.12 & 6 & Quench Jastec \\
\hline 10 & 6.05 & 5.95 & Quench Bruker \\
\hline 20 & 6.05 & 5.91 & Quench Jastec \\
\hline 51 & 6.04 & 5.86 & Quench Bruker \\
\hline 100 & 6.03 & 5.85 & Quench Jastec \\
\hline 200 & 6.02 & 5.82 & Quench Bruker \\
\hline 500 & 6.01 & 5.81 & Quench Jastec \\
\hline 1000 & 5.98 & 5.77 & Quench Bruker \\
\hline
\end{tabular}

There is a slight difference in the performance drop between the first and $1000^{\text {th }}$ cycle. While $T_{\text {cs }}$ in TFIO6 (the conductor that did not experience initial quenches) drops by only $0.21 \mathrm{~K}$ (Jastec) and $0.32 \mathrm{~K}$ (Bruker), in the TFIO7 (intermediate $10 \mathrm{DC}$ tests followed by sample quenches) $T_{\mathrm{cs}}$ drops slightly more, namely by $0.27 \mathrm{~K}$ (Jastec) and $0.40 \mathrm{~K}$ (Bruker).

In addition, once the DC tests start to be performed on TFIO6 between the cycles no. 1000 and 1067, $T_{\mathrm{cs}}$ drops an order of magnitude more than what would correspond to just pure $67 \mathrm{EM} \mathrm{cy}-$ cles. The $T_{\text {cs }}$ drops by $0.04 \mathrm{~K}$ (Jastec) and $0.10 \mathrm{~K}$ (Bruker), while the expected performance drop in both Jastec and Bruker is only around $0.005 \mathrm{~K}$ per 67 cycles. (The expected $T_{\mathrm{cs}}$ drop is extrapolated from the difference between $T_{\mathrm{cs}} \# 500$ and $T_{\mathrm{cs}} \# 1000$ in Table 2.) This clearly shows that the performed DC tests with the fast dump slightly, but observably, degraded $T_{\mathrm{cs}}$.

Also the $n$-values measured in the $I_{\mathrm{c}}$ runs seem to support the conclusion that sample TFIO7 has degraded more than the TFIO6 one, see Table 3. However, it has to be noted that we did not perform an initial $I_{\mathrm{c}}$ measurement for the TFIO6 sample (not to risk a voltage take-off in the initial phase of testing), and therefore we can only compare the $n$-values at 1001 cycles, and not the relative change between e.g. the $3^{\text {rd }}$ and the $1001^{\text {st }}$ cycle.

Table 3 The $n$-values of the TFIO6 and TFIO7 samples.

\begin{tabular}{|c|c|c|c|c|}
\cline { 2 - 5 } \multicolumn{1}{c|}{} & \multicolumn{2}{c|}{ TFIO 6 } & \multicolumn{2}{c|}{ TFIO 7 } \\
\cline { 2 - 5 } \multicolumn{1}{c|}{} & Jastec & Bruker & Jastec & Bruker \\
\hline Cycle \# & n-value & n-value & n-value & n-value \\
\hline 3 & - & - & 11.2 & 12.4 \\
\hline 1001 & 9.7 & 12.3 & 8.3 & 10.6 \\
\hline 1068 & 9.4 & 11.3 & - & - \\
\hline 1070 & 9 & 10.6 & - & - \\
\hline
\end{tabular}

\section{DISCUSSION}

We have presented three independent observations that demonstrate enhanced (or accelerated) degradation of $T_{\mathrm{cs}}$ due to voltage take-off (quench) followed by the very fast current discharge. Very recently, a difference in $T_{\mathrm{cs}}$ degradation has been reported in section 6.2 of [6], where a concept of "conductor conditioning" or "training" was introduced in order to explain the difference in the rate of degradation between the ITER TF conductors of the same manufacturer. By comparing the $T_{\mathrm{cs}}$ evolution in TFIO1 and TFI-SULTAN (TF insert-coil sample tested in SULTAN, whose $T_{\mathrm{cS}}$ degraded more than that of TFIO1), it was found that "the degradation of the conductor seems to decrease when the conductor is exposed to lower EM loads at the beginning of the testing series before being exposed to the highest EM loads (i.e. a training effect)."

However, also in this case an (alternative) explanation to the different $T_{\mathrm{cs}}$ degradation could be related to the sample quenches. While TFI sample was tested in a usual way with sample quenches, quenches were avoided during TFIO1 testing.

What can be the underlying mechanism causing the quenchrelated degradation? The typical highest temperature observed during the sample quenches in SULTAN is around $25 \mathrm{~K}$. The temperature sensors are located on the steel jacket upstream and downstream of the quenched region (the quench is generally initiated in a $450 \mathrm{~mm}$ long high-field zone), and therefore the hotspot temperature in the cable might marginally exceed $25 \mathrm{~K}$. The degradation due to the thermal expansion of either strands or jacket is therefore hard to believe. It is also unlikely that local $\mathrm{AC}$ currents induced by fast current dump could be too high to potentially damage the strands, e.g. by locally induced forces. 
The conductor loses its superconducting properties during quench, the resistance of the coupling current loops becomes orders of magnitude higher than in the superconducting state, which substantially suppresses the induced AC loss currents.

We have not found a difference between the degradation of the conductor section, in which the voltage take-off was induced, compared to the second leg. This suggests that the conductor degradation is due to the fast current dump rather than due to the voltage take-off.

In conclusion, we are lacking a solid plausible explanation for the suspected degradation. One might speculate about some loadrate dependent effects in a cable behaving as a non-elastic spring, in which fast varying load induce more total displacement than a slow varying one.

\section{CONCLUSIONS}

The $T_{c s}$ degradation of ITER TF conductors has evolved into a very complex phenomenon. It clearly depends on the electromagnetic and thermal cycling, and we seem to have some evidence now that it is enhanced also by sample quenches followed by fast current dumps. All these individual factors degrade DC performance of the cable, however the degradation gets clearly increased when these factors act in combination. It even seems that the degradation can be limited by an appropriately chosen sequence of partial EM load and thermal cycling in the initial phase of the coil operation, i.e. by the so-called conditioning/training [6].

The good news for the ITER project is that the reported degradation due to the sample quenches followed by the fast current discharge will not happen in the ITER TF coils that will be under all circumstances discharged slowly $\left(\tau_{\text {discharge }}=11 \mathrm{~s}\right)$, even in the case of a real quench. The $T_{c s}$ degradation observed in SULTAN samples and in TF Insert Coil tests are therefore likely slightly overestimating the degradation that we might see during the ITER TF coil operation.

On the other hand, the observation that the voltage take-off (an initial phase of a quench) with the fast current discharge degrade the ITER TF conductor came unexpected. The underlying mechanism is not understood, and we may wonder what will happen in case of a real, fully-developed quench in the ITER TF magnet, when the hot-spot temperature in the cable might reach $250 \mathrm{~K}$ during the transient. Surprises in terms of $T_{c s}$ degradation are not excluded, as no quench test with a fully developed temperature rise has ever been performed on the fullsize ITER conductor due to non-existing test facility able to do so.

\section{ACKNOWLEDGMENT}

The views and opinions expressed herein do not necessarily reflect those of the ITER Organization. The technical support of Paul Scherrer Institute is highly appreciated.

\section{REFERENCES}

[1] M. Breschi et al., "Results of the TF conductor performance qualification samples for the ITER project", Supercond. Sci. Technol. 25, 2012, Art. no. 095004

[2] M. Breschi, D. Macioce and A. Devred, "Performance analysis of the toroidal field ITER production conductors", Supercond. Sci. Technol. 30, 2017, Art. no. 055007.

[3] A. Devred et al., "Challenges and status of ITER conductor production", Supercond. Sci. Technol. 27, 2014, Art. no. 044001.

[4] A. Devred et al., "Status of Conductor Qualification for the ITER Central Solenoid", IEEE Trans. Appl. Supercond. 23, 2013, Art. no 6001208.

[5] D. Besette, "Design of a $\mathrm{Nb}_{3} \mathrm{Sn}$ Cable-in-Conduit Conductor to Withstand the 60000 Electromagnetic Cycles of the ITER Central Solenoid," IEEE Trans. Appl. Supercond., vol. 24, no. 3, 2014, Art. no. 4200505.

[6] N. Mitchell, M. Breschi, V. Tronza, "The use of Nb3Sn in fusion: lessons learned from the ITER production including options for management of performance degradation," Supercond. Sci. Technol. 33, 2020, Art. no. 054007.

[7] C. Sanabria, P. J. Lee, W. Starch, A. Devred, and D. C. Larbalestier, "Metallographic autopsies of full-scale ITER prototype cable-in-conduit conductors after full cyclic testing in SULTAN: III. The importance of strand surface roughness in long twist pitch conductors", Supercond. Sci. Technol. 29, 2016, Art. no. 074002.

[8] D. Yue, X. Zhang, and Y. H. Zhou "Buckling Behavior of $\mathrm{Nb}_{3} \mathrm{Sn}$ Strand Caused by Electromagnetic Force and Thermal Mismatch in ITER CableIn-Conduit Conductor", IEEE Trans. Appl. Supercond., vol. 27, no. 7, 2017, Art. no. 8400911 .

[9] S. A. March et al., "Effect of Thermal Loading on Nb3Sn CICC Performance", IEEE Trans. Appl. Supercond., vol. 22, no. 3, 2012, Art. no. 4803604.

[10] H. Ozeki, et al. " $T_{c s}$ Measurement Result of ITER Toroidal Field Insert Coil Tested in 2016", IEEE Trans. Appl. Supercond., 2018, in press.

[11] P. Bruzzone, A. Anghel, A. Fuchs, G. Pasztor, B. Stepanov, M. Vogel and G. Vecsey, "Upgrade of operating range for SULTAN test facility", IEEE Trans. Appl. Supercond. 12, 2002, 520-523.

[12] H. Ozeki et al., "Manufacture and Quality Control of Insert Coil With Real ITER TF Conductor", IEEE Trans. Appl. Supercond., vol. 26, no. 4, 2016, Art. no 4202504.

[13] T. Ando and H. Tsuji, "ITER central solenoid (CS) model coil project", TEION KOGAKU (J. Cryogenics Supercond. Soc. Jpn), vol. 36, no. 6, pp. 309-314, 2001. 